\title{
8 Purifying the ykkCD Mutant Toxin Sensor RNA and Evaluating its Purity Using Denaturing PAGE and UV spectrometry
}

\subsection{Learning Objective}

The purpose of this lab is to learn how to purify RNA samples and evaluate their purity using a denaturing polyacrylamide gel electrophoresis (urea PAGE). RNA molecules synthesized in vitro transcription are purified to remove the RNA polymerase, the DNA template and unused nucleotides (NTPs). The mutant toxin sensor RNA has to be purified for the following reasons: (1) The DNA template and unused nucleotides can interfere with accurate determination of RNA concentration using UV spectrometry, because these compounds also absorb the UV light at $260 \mathrm{~nm}$. (2) The RNA polymerase, transcription buffer and unused NTPs may interfere with subsequent binding assays.

\subsection{Mini Project Flowchart}

The bolded block in the flowchart below highlights the role of the current experiment in the mini project.

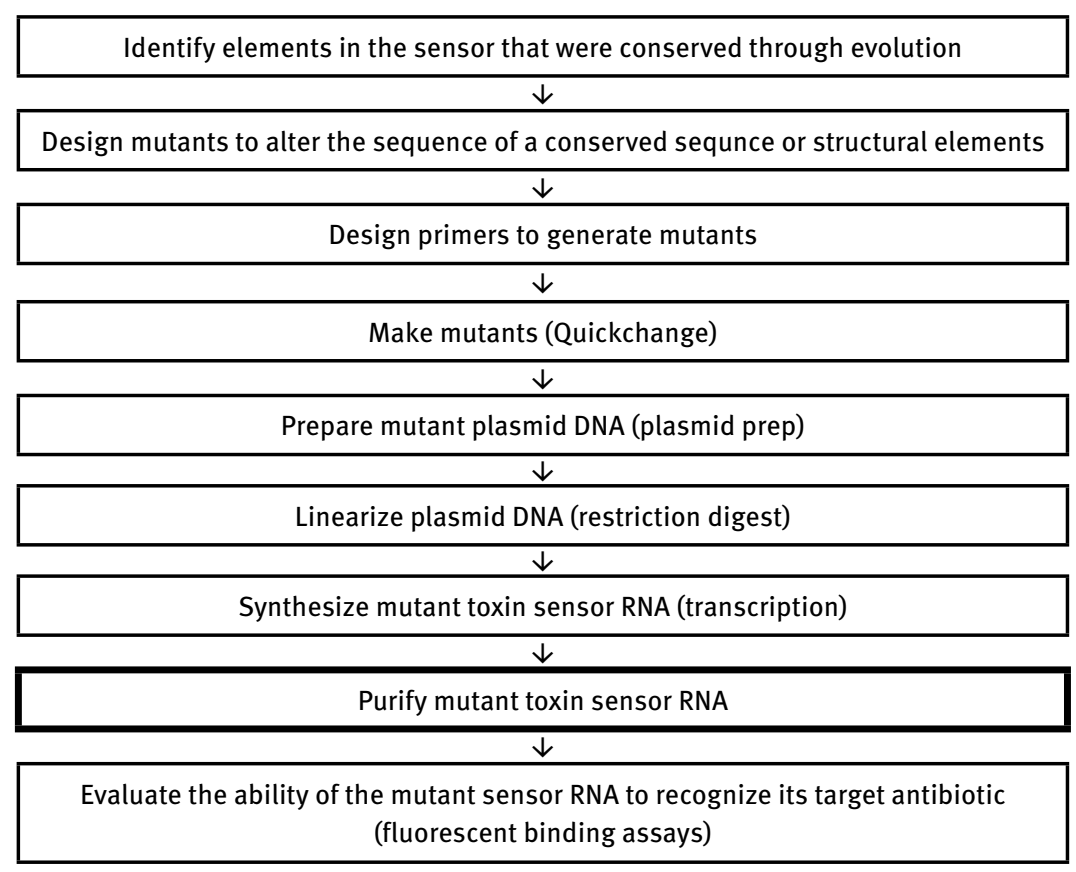




\subsection{RNA Purification Methods}

In vitro synthesized RNAs can be purified using various methods: denaturing polyacrylamide gel electrophoresis (denaturing PAGE), phenol/chloroform extraction or column purification. A brief description of each method is provided below.

\subsection{Denaturing PAGE}

Denaturing PAGE separates RNA molecules by their molecular weight (Fig. 8.1A). This method is used for both RNA purification and evaluation of RNA purity. In the latter case, RNAs are visualized using EtBr staining. Denaturing PAGE of RNAs is similar to agarose gel electrophoresis of DNAs. RNAs also migrate in the medium due to their negative charge from the negative to the positive electrode. There are two main differences: (1) the separation medium is polyacrylamide instead of agarose and (2) RNA samples are denatured so that the rate of migration in the gel is only affected by molecular weight and not by RNA structure. Since RNAs fold into stable, complex structures, to separate RNAs by molecular weight RNA molecules have to be denatured. Denaturation is achieved using urea - a chaotrop that competes with nucleobases for H-bonding thereby unfolding the RNA molecule (Fig. 8.1B). Polyacrylamide forms a similar net-like porous structure as agarose, but the pore size is significantly smaller accommodating separation of smaller nucleic acids.

A

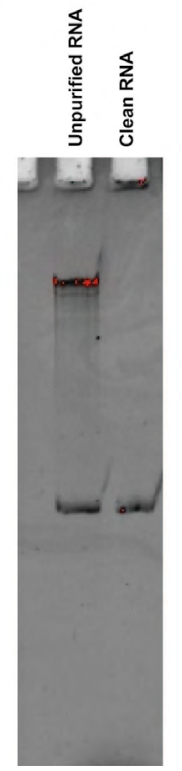

B<smiles>NC(N)=O</smiles>

Figure 8.1: Denaturing polyacrylamide electrophoresis of RNAs. (A) RNA molecules are separated by molecular weight. The DNA template in the unpurified sample migrates on top, because of its larger molecular weight. (B) Urea is a chaotrop that unfolds RNAs by $\mathrm{H}$-bonding with nucleobases. 
Polyacrylamide gel is a polymer of acrylamide and methylenebisacrylamide; the methylenebisacrylamide is responsible for the crosslinks between acrylamide chains yielding a porous structure. The crosslinking ratio (ratio between acrylamide and methylenebisacrylamide) determines the pore size of the medium. A higher crosslinking ratio denotes a medium with a smaller pore size. That is designed to separate smaller RNAs (Fig. 8.2).

Acrylamide Methylenebisacrylamide<smiles>C=CC(=O)NCNC(=O)C=C</smiles>

$$
\downarrow
$$<smiles>CCC(CC)CC(CC(C)C(N)=O)C(=O)NCNC(=O)C(CC(CC(C)C(N)=O)C(N)=O)CC(CC(CC(CC(CC)C(N)=O)C(N)=O)C(N)=O)C(N)=O</smiles>

Figure 8.2: Polyacrylamide gel electrophoresis. The polyacrylamide medium is generated by freeradical polymerization. The ratio of acrylamide: methylenebisacrylamide determines the pore size of the gel.

Denaturing PAGE produces the cleanest RNA. The transcription mixture is separated on a denaturing PAGE. The desired RNA band (toxin sensor RNA) is excised (cut) from the gel and the RNA is eluted. This method removes the RNA polymerase, the DNA template, unused nucleotides and any in vitro synthesized RNAs that differ in size from the toxin sensor RNA (mostly RNAs that were the product of degradation). Denaturing PAGE purification of RNA is time consuming and only used when highpurity RNA is an absolute requirement for structure determination, time-resolved fluorescent spectroscopy etc. 


\subsection{Phenol/chloroform Extraction}

Phenol/chloroform extraction removes the polymerase and most unused nucleotides from the reaction mixture. Since some unused nucleotides and the DNA template is still in the mixture, accurate determination of RNA concentration is not possible using this purification method (recall, all of these nucleic acids absorb the UV light at $260 \mathrm{~nm}$ ). To evaluate the ability of the mutant toxin sensor RNA to recognize the antibiotic tetracycline, the binding affinity between the mutant sensor and tetracycline has to be determined accurately. Since binding affinity measurements require precise knowledge of RNA concentration, phenol/chloroform extraction does not produce adequate RNA quality for our purposes.

\subsection{Column Purification}

Our method of choice for RNA purification is column purification. This method is very similar to purification of plasmid DNAs during plasmid preparation. When RNA is synthesized in vitro for biochemical purposes, the goal is to get high concentrations of the target RNA in water. In contrast the RNA after synthesis is contaminated with the polymerase, the DNA template, unused nucleotides and a buffer. During column purification all of these contaminants are removed. Column purification of RNAs utilize the fact that in vitro synthesized RNAs are highly negatively charged (compared to proteins) and fairly small (compared to the template DNA). Column purification is advantageous; because it is quick, it removes the DNA template and unused nucleotides, thus enables accurate determination of RNA concentration.

Make sure to follow the protocol exactly as written. Always wear gloves and store RNA on ice to prevent degradation of your sample.

The exact protocol of RNA purification is dependent on the kit used and will be provided in the lab. A general outline of procedure is presented below.

1. Column purification of RNA usually starts with binding the transcription mixture to an anion exchange column. This separation method based on the difference in charge between protein, nucleotides and nucleic acids (polymers of nucleotides).

2. The template DNA and the RNA binds to the column, while unincorporated nucleotides (too small) and the polymerase (not charged enough) flow through.

3. The DNA template is much larger (more negatively charged) than the synthetic RNA and this means it is not eluted from the column. 


\section{What are we going to do?}

Today we will purify the in vitro synthesized toxin sensor RNA via column purification then evaluate RNA purity using denaturing urea PAGE and determine RNA concentration using UV spectrometry.

\section{PROTOCOLS}

Reagents and equipment needs are calculated per six student teams. There is $\sim 20 \%$ excess included.

\section{Equipment/glassware needed}

1. Six disposable test tubes at least $10 \mathrm{ml}$ volume

2. Three sets of micropipettes 20-100 $\mu \mathrm{l}$ and 2-20 $\mu \mathrm{l}$

3. Three sets of mini protean 3 PAGE apparatus

4. Power supply (to run urea PAGE)

5. UV spectrometer

6. Gel documentation system

7. Twenty $1.5 \mathrm{ml}$ centrifuge tubes (Eppendorf)

8. Heating block (to boil samples)

\section{Solutions needed}

1. $311 \times$ TBE (Tris-borate-EDTA) $\mathrm{pH}=8.0$

2. $30 \mu \mathrm{l} 1 \% \mathrm{EtBr}$

3. $300 \mu$ urea gel loading dye (2x); (6 M urea, $0.5 \mathrm{x}$ TBE, $1 \%$ bromophenol blue)

4. Urea gel solution mix; (6 M urea, 0.5 x TBE, 10\% acrylamide: bis acrylamide 19:1 crosslinking ratio)

5. $1 \mathrm{ml} \mathrm{10 \%} \mathrm{ammonium} \mathrm{persulfate} \mathrm{(APS)} \mathrm{freshly} \mathrm{made}$

6. $50 \mu \mathrm{l}$ TEMED

\section{Column purification of RNA}

Protocol depends on the kit used and will be provided in the lab. See the top of the page for a basic overview.

\section{Determine RNA concentration using UV spectroscopy}

RNA concentration is determined in a similar manner as DNA concentration. RNAs also absorb at $260 \mathrm{~nm}$ due to the heterocyclic aromatic nucleobases. To calculate RNA concentration the Beer-Lambert law is used: $A(@ 260 \mathrm{~nm})=\varepsilon^{\star} \mathrm{C}^{\star} l$ where $\mathrm{l}=1 \mathrm{~cm}, \mathrm{c}$ is the RNA concentration in mole/liter and $\varepsilon=1149800$ for the ykkCD toxin sensor RNA.

\section{Denaturing PAGE of RNA}

Make the gel

1. Assemble gel plates and check them for leaking with EtOH.

2. Pipette $10 \mathrm{ml}$ of urea gel mixture into a test tube. 
3. Add $100 \mu \mathrm{l} 10 \%$ APS and $10 \mu \mathrm{l}$ TEMED to initiate polymerization.

4. Mix well and pour in between glass plates. Place in comb. WATCH OUT, THIS IS QUICK! WAIT UNTIL GEL POLYMERIZES!

5. Once gel is polymerized assemble gel gasket.

6. Pour $0.5 \mathrm{x}$ TBE running buffer into gasket.

7. Wash wells.

8. Pre-run gel for $30 \mathrm{~min}$ at $15 \mathrm{~W}$. This step gets rid of any acrylamide that did not form a polymer and any free radical produced during polymerization.

Prepare sample and run the gel. Run $10 \mu$ unpurified and $10 \mu l$ purified RNA on the gel.

1. Mix $10 \mu \mathrm{l}$ RNA with equal volume of urea dye (6M urea, $0.5 \mathrm{x}$ TBE, $1 \%$ bromophenol blue).

2. Boil samples for $5 \mathrm{~min}$ in the heating block. Quick spin samples.

3. Load samples onto the gel. REMEMBER WHERE YOU LOADED YOUR SAMPLE!

4. Run gel for $15 \mathrm{~min}$ at $15 \mathrm{~W}$ or until the dye reaches the bottom of the gel.

5. Place the gel into $50 \mathrm{ml}$ staining solution (50 ml TB buffer and $10 \mu \mathrm{l} \mathrm{EtBr}$ ) for $10 \mathrm{~min}$.

6. Scan gel.

\section{Precautions}

1. Acrylamide is a neurotoxin

2. EtBr is a carcinogen/mutagen

MAKE SURE TO WEAR GLOVES

Note to the instructor

The experiment in Chapter 8 is designed to purify the ykkCD toxin sensor RNA, but the protocol with minor modifications could be used for any other RNA molecule. Due to the time requirements of making and pre-running a denaturing PAGE it is recommended to start the laboratory session with making the gel then purifying the RNA while the gel pre-runs. Qiagen nucleotide removal kit and GeneJet RNA Cleanup kit (Thermo Scientific) were used by the authors, but similar kits from alternate vendors may also be used. It is preferable that each student team make and run a denaturing PAGE to increase the educational value of the laboratory, but if supplies are limited six student teams may share a gel. The denaturing PAGE may also be made by teaching assistant or the instructor ahead of time. In this case however the laboratory is more appropriate to be called a demonstration of RNA purification. 


\section{Prelab Questions for RNA Purification}

1. What is "urea gel electrophoresis (denaturing PAGE)"?

$/ 2$ pts

2. What do you remove from the RNA sample during RNA purification? Name two things.

$/ 2 \mathrm{pts}$

3. What type of precautions do you have to observe when handling acrylamide? Explain your answer.

$/ 3$ pts

4. To run a denaturing PAGE you need $1 \mathrm{~L} 0.5 \mathrm{x}$ TBE buffer. The stock solution is $10 \mathrm{x}$ TBE. Explain how you make your buffer (show your calculation). 


\section{RNA Purification}

\section{Lab Report Outline and Point Distribution}

1. Several sentences defining the goal/purpose of each procedure. Make sure you clearly indicate the role of this step in the mini project (5 pts.).

2. Briefly describe the methods available for RNA purification. Indicate why we choose column purification in the lab (7 pts.).

3. Draw a flowchart of the RNA purification. Make sure to indicate the name of each buffer used and the volume of buffer needed (10 pts.).

4. Include a picture of your denaturing PAGE gel scan with your samples marked (4 pts.).

5. Evaluate the purity of your mutant ykkCD sensor RNA by comparing the bands before and after purification. Explain your answer (5 pts.).

6. Report the concentration of your purified ykkCD RNA. Make sure you use the Beer-Lambert law and the molar extinction coefficient provided to carry out your calculation. Show your work (5 pts.).

7. Calculate ykkCD sensor RNA yield. Show your work (5 pts.).

8. Report the 260/280 absorbance-ratio of the purified ykkCD RNA. Evaluate RNA purity based on this value. What types of contaminants could cause a peak around 180 wavelength (5 pts.)?

9. Troubleshooting question: Your colleague performed RNA purification with the same kit you used. He later performed a binding assay and determined that his ykkCD RNA (wild type) had low affinity for tetracycline. His denaturing PAGE and 260/280 absorbance values each indicated clean RNA and he appeared to have good yield. He later determined that his RNA solution contained ethanol. In your judgment, which step of the purification did he make the mistake? Is there any way to rescue his RNA (4 pts.)? 